\title{
Evolution saisonnière du flux de sève dans un peuplement de pins maritimes *
}

\author{
D Loustau 1**, A Granier 2, F El Hadj Moussa 1
}

\author{
Collaboration technique de M Sartore, M Guedon \\ 1 Station INRA de recherches forestières, laboratoire d'écophysiologie et nutrition, \\ domaine de l'Hermitage, Pierroton, 33610 Cestas; \\ 2 INRA, centre de recherches forestières de Nancy Champenoux, \\ laboratoire de bioclimatologie et d'écophisiologie, 54280 Seichamps, France
}

(Reçu le 20 janvier 1990; accepté le 14 août 1990)

\begin{abstract}
Résumé - Le débit de sève d'un échantillon de pins maritimes a été suivi en continu au cours de trois saisons de végétation dans un peuplement adulte. Au printemps et au début de l'été, quand le pourcentage d'eau extractible (PEE) est supérieur à $20 \%$, les fluctuations journalières du débit de sève suivent celles de l'ETP. La densité de flux de sève journalière varie entre 15 et $30 \mathrm{~kg}^{\circ} \mathrm{dm}^{-2} \cdot \mathrm{j}^{-1}$. Le potentiel de base varie entre -0.3 et $-0.5 \mathrm{MPa}$ et la conductance stomatique présente des cinétiques journalières décroissantes de $0.3 \mathrm{~cm} \cdot \mathrm{s}^{-1}$ le matin à $0.1 \mathrm{~cm} \cdot \mathrm{s}^{-1}$ l'après-midi. Au cours de l'été, lorsque le PEE diminue sous le seuil de $20 \%$, le débit de sève descend entre 5 et $10 \mathrm{~kg}^{\circ} \mathrm{dm}^{-2} \mathrm{oj}^{-1}$ et ses variations sont surtout liées aux épisodes pluvieux. Le potentiel de base diminue de -0.6 a -1.2 $\mathrm{MPa}$ selon le niveau hydrique du sol et la conductance stomatique est divisée par 5 . Le potentiel hydrique minimal, mesuré en milieu de journée, s'est maintenu autour de $-1.6 \mathrm{MPa}$, indépendamment des conditions d'humidité du sol.
\end{abstract}

\footnotetext{
"Etude réalisée dans le cadre de I'AIP «Fonctionnement hydrique du pin maritime» (INRA) et de I'ATP.PIREN. «Influence à l'échelle régionale des couvertures pédologiques et végétales sur les bilans hydriques et minéraux du sol».

** Correspondance et tirés à part.

Abréviations : $A$, énergie disponible (Watts $\left.\bullet \mathrm{m}^{-2}\right)$; $C p$, chaleur spécifique de l'air $\left(\mathrm{J} \cdot \mathrm{kg}^{-1} \cdot \mathrm{K}^{-1}\right) ; D S A T$, déficit de saturation de l'air en vapeur d'eau $(\mathrm{Pa}) ; d T_{h}$, écart thermique instantané observé entre sonde chaude et sonde froide $\left({ }^{\circ} \mathrm{C}\right) ; d T_{m}$, écart thermique maximal journalier $\left({ }^{\circ} \mathrm{C}\right) ; E T P$, évapotranspiration $\left(\mathrm{mm} \cdot \mathrm{h}^{-1}\right) ; g_{a}$, conductance aérodynamique $\left(\mathrm{m} \cdot \mathrm{s}^{-1}\right) ; g_{s}$, conductance stomatique $\left(\mathrm{cm} \cdot \mathrm{s}^{-1}\right) ; h$, hauteur moyenne du couvert (13 m); $J_{s}$, densité de flux de séve par unité de surface conductrice à $130 \mathrm{~cm}\left(\mathrm{en} \mathrm{kg}^{\circ} \cdot \mathrm{dm}^{-2} \mathrm{oj}^{-1}\right) ; J_{s C}$ densité de flux moyenne du peuplement à $130 \mathrm{~cm}\left(\mathrm{en} \mathrm{kg}^{\circ} \cdot \mathrm{dm}^{-2} \cdot \mathrm{j}^{-1}\right) ; J$, flux de sève $\left(\mathrm{kg}^{\circ} \mathrm{h}^{-1}\right) ; k$, constante de Von Karman $\left(0.41\right.$, sans dimension); $L_{s}$, conductance hydraulique spécifique $\left(\mathrm{kg}^{\circ} \mathrm{dm}^{-2} \cdot \mathrm{h}^{-1} \cdot \mathrm{MPa}^{-1}\right) ; P E E$, contenu du sol en eau extractible par la végétation, exprimé en pourcentage de la réserve totale $(\%) ; R g$, rayonnement global (Watts $\left.\cdot \mathrm{m}^{-2}\right) ; R$, stock mesuré $(\mathrm{mm}) ; \boldsymbol{R}_{m}$, stock minimum (mm); $\boldsymbol{R}_{c}$ stock à la capacité au champ (mm); $s$, dérivée de la fonction reliant la pression de vapeur saturante à la température $\left(\mathrm{Pa}^{\circ} \mathrm{K}^{-1}\right) ; S A$, section d'aubier à la hauteur du point d'insertion des sondes $\left(\mathrm{dm}^{2}\right) ; S A_{c}$ section d'aubier totale du peuplement à $130 \mathrm{~cm}$ (en $\left.\mathrm{dm}^{2} \cdot \mathrm{ha}^{-1}\right)$; STE, surface terrière $\left(\mathrm{dm}^{2}\right) ; T r$, transpiration journalière $\left(\mathrm{mm} \cdot \mathrm{j}^{-1}\right) ; U_{z^{\prime}}$ vitesse du vent à la cote $\mathrm{z}\left(\mathrm{m} \cdot \mathrm{s}^{-1}\right) ; \gamma$, constante psychrométrique $\left(\mathrm{Pa} \cdot \mathrm{K}^{-1}\right) ; \lambda$, chaleur latente de vaporisation de l'eau $\left(\mathrm{J} \cdot \mathrm{kg}^{-1}\right) ; \psi_{b}$, potentiel hydrique de base des aiguilles (MPa); $\psi_{s p}$ potentiel hydrique a linterface racine-sol (MPa); $\psi_{t}$ potentiel hydrique foliaire (MPa); $\psi_{o}$, potentiel hydrique à densité de flux nulle $(\mathrm{MPa}) ; \rho$, densité de l'air sec $\left(\mathrm{kg} \cdot \mathrm{m}^{-3}\right)$.
} 
La sécheresse édaphique a réduit le débit de sève d'une part en réduisant la différence de potentiel sol-feuille et d'autre part, en provoquant une diminution de la conductance hydraulique globale, qui est divisée par 3.

potentiel hydrique follaire / conductance hydraulique / déficit hydrique du sol / conductance stomatique / transpiration

Summary - Seasonal varlations of sap flow in a maritime pine stand. The sap flow density of a sample of trees was measured continuously throughout three growing seasons, in a mature stand of maritime Pine. In spring and early summer, the relative extractable water content of soil (REWC) was always greater than 20\%. The sap flow density varied between 15 and $30 \mathrm{~kg} \cdot \mathrm{dm}^{-2} \mathrm{j}^{-1}$ and daily variations of sap flow followed the fluctuations of the potential evapo-transpiration. The daily course of stomatal conductance showed a decrease from $0.3 \mathrm{~cm} \cdot \mathrm{s}^{-1}$ in the morning to $0.1 \mathrm{~cm} \cdot \mathrm{s}^{-1}$ in the afternoon. Predawn water potential of neodles remained between -0.3 and $-0.5 \mathrm{MPa}$. From mid-summer to the end of the season, the REWC fell below $20 \%$ and sap flow decreased 4-fold. Daily variations of sap flow were mainly related to rainfall. Stomatal conductance was reduced to $0.05 \mathrm{~cm}^{\circ} \mathrm{s}^{-1}$ and predawn water potential fell to between -0.6 and 1.12 MPa, according to the soil water content. Throughout the season, the lowest daily values of water potential were maintained around - 1.16 MPa. Soil water deficit appeared to reduce the sap flow density by decreasing the difference in water potential between the soil and the leaves, and the bulk hydraulic conductivity along this circuit by a factor of three.

needle water potentlal / hydraullc conductance / soil water deficlt / stomatal conductance / transpiration

\section{INTRODUCTION}

Pour comprendre les facteurs qui contrôlent le flux hydrique qui traverse un arbre, il est nécessaire de situer celui-ci dans le continuum sol-plante-atmosphère (Running et al, 1975).

Le flux transpiratoire, en phase vapeur, dégagé à partir de la surface foliaire d'un arbre dépend de facteurs climatiques et peut être décrit, à partir de données micrométéorologiques, par la relation de Penman-Monteith. Le contrôle stomatique est le principal facteur de régulation du flux transpiratoire des arbres forestiers; de nombreux travaux mentionnent les effets de facteurs climatiques, rayonnement lumineux et déficit de saturation de l'air en vapeur d'eau, ou édaphiques (déficit hydrique du sol), sur la conductance stomatique (Sandford et Jarvis, 1986; Schulze et al, 1987; Sharkey et Ogawa, 1987) et leurs conséquences sur la transpiration (Jarvis et McNaughton, 1985).
Le flux de sève qui s'établit entre le système racinaire et les surfaces foliaires transpirantes, peut être décrit par des équations de transfert, suivant une analogie électrique. II dépend à la fois de l'état hydrique des surfaces transpirantes, de la disponibilité de l'eau dans le sol et des propriétés hydrauliques (conductivité, capacité) des différentes parties du réseau conducteur de sève. L'état hydrique des surfaces transpirantes et la disponibilité de l'eau dans le sol sont définis respectivement par le potentiel hydrique foliaire et le potentiel hydrique du sol. Ces deux variables fixent les conditions aux limites du transfert de la sève.

Au cours d'une sécheresse édaphique, l'ensemble des paramètres qui contrôlent le flux hydrique dans sa phase liquide ou dans sa phase vapeur est modifié. II est classique d'observer une réduction de la conductance stomatique (Schulze et al, 1987), et une chute du potentiel hydrique de base (Aussenac et Granier, 1978). Une 
diminution de la conductivité hydraulique globale du circuit sol-feuille est également observée par certains auteurs (Running, 1980; Pena et Grace, 1986; Granier et al, 1988).

II existe relativement peu d'études proposant une appréhension synthétique du fonctionnement hydrique de l'arbre dans le continuum sol-plante-atmosphère et qui prennent en compte à la fois les mécanismes qui contrôlent le flux hydrique dans sa phase liquide et ceux qui interviennent en phase vapeur. Seule cette approche permet pourtant de définir les contraintes hydriques subies par les arbres en conditions naturelles, et de décrire les modifications qu'elles entraînent dans leur fonctionnement hydrique.

Le but de cet article est de montrer l'effet des principaux facteurs abiotiques (contenu en eau du sol, paramètres climatiques) sur l'évolution saisonnière du débit de sève, de la résistance stomatique et de la transpiration du pin maritime. En dépit de son importance économique et écologique dans le Sud-Ouest européen, où cette essence couvre plus de 3,5 millions d'hectares, le fonctionnement hydrique du pin maritime n'a été abordé que de façon fragmentaire (Guyon et Kremer, 1982; Granier et al, 1989; Loustau et El Hadj Moussa, 1989). Le déficit estival en eau du sol est pourtant observé constamment dans cette région et sur pratiquement toute l'aire de cette espèce (Choisnel et al, 1987; Baradat, 1988). Par ailleurs, différents travaux (Aussenac et al, 1982; Donner et Running, 1986; Linder et al, 1987) montrent que des pratiques sylvicoles courantes pour le pin maritime, comme l'éclaircie ou la fertilisation, modifient le fonctionnement hydrique des arbres et des peuplements.

Le débit de sève d'un échantillon de pins a été suivi pendant trois saisons consécutives de croissance dans un peuplement adulte. Ces mesures ont été complé- tées par des mesures de conductance stomatique, de potentiel hydrique foliaire et de contenu en eau du sol, à partir desquelles les mécanismes de régulation des flux hydriques chez le pin maritime et leurs effets sont discutés.

\section{MATÉRIEL ET MÉTHODES}

\section{Site expérimental}

Le régime climatique de la zone expérimentale est de type océanique, avec des hivers doux et pluvieux et des étés relativement secs; les moyennes des totaux annuels (1951-1980) de température et de pluviométrie sont de $12.5^{\circ} \mathrm{C}$ et $932 \mathrm{~mm}$.

La parcelle expérimentale est située à $20 \mathrm{~km}$ au Sud-Ouest de la ville de Bordeaux (latitude $44^{\circ} 42^{\prime}$ Nord, longitude $0^{\circ} 46^{\prime}$ Ouest), en aLande humide", unité écologique régionale caractérisée par une nappe phréatique fluctuant annuellement entre 0 et $150 \mathrm{~cm}$ de profondeur, un sol podzolique sableux à horizon $B$ induré, et une végétation spontanée dominée par la Molinie (Molinia coerulea Moench). Elle couvre une superficie de 16 ha.

Le développement des systèmes racinaires des arbres et de la Molinie est limité en protondeur par la présence de l'horizon $B$ induré qui constitue un obstacle à la fois structural et chimique (teneur en aluminium élevée) à la pénétration racinaire.

Le peuplement de pin, conduit selon les normes de sylviculture intensive, provient d'un semis en ligne de 1970; en 1988, la densité est de 880 tiges $/$ ha, la circonférence et la hauteur moyenne respectivement de $60 \mathrm{~cm}$ et de $12 \mathrm{~m}$ (classe I des tables de production de Decourt et Lemoine, 1969); l'espacement des lignes est de $4 \mathrm{~m}$, la distance moyenne entre deux tiges sur une ligne est de $3 \mathrm{~m}$.

La surface foliaire développée est estimée entre 6 et $7 \mathrm{~m}^{2 /} \mathrm{m}^{2}$ de surface au sol, ce qui correspond à un indice foliaire peu différent de 3 (El Hadj Moussa, 1989).

L'ensemble des mesures a été réalisé au sein d'un placeau expérimental de $1200 \mathrm{~m}^{2}$, 
situé au centre de la parcelle, afin de minimiser les effets de bordure.

L'expérimentation comprend trois périodes de mesure : 1987, 1988 et 1989 . En 1987, la pluviométrie et I'ETP mensuelles entre mars et novembre ont été peu différentes des normales. L'année 1988 a été marquée par un excédent pluviométrique important, l'année 1989 s'est au contraire singularisée par un déficit remarquable.

\section{Mesures de flux de sève}

\section{Principe}

La méthode de mesure utilisée est décrite par Granier $(1985,1987)$. Son principe repose sur le fait que l'écart de température entre deux sondes insérées dans le xylème d'un arbre, l'une dégageant un flux de chaleur constant par effet Joule, l'autre étant en équilibre avec la température du xylème, dépend de la vitesse de circulation de la sève dans le xylème. Une formule empirique établie à partir de mesures opérées en laboratoire donne :

$$
J_{s}=4,284\left(\frac{d T_{m}-d T_{h}}{d T_{h}}\right)^{1,231}
$$

A partir de cette mesure, il est possible de calculer le flux de sève total traversant le tronc d'un arbre par la relation :

$$
J=J_{s} \times S A
$$

\section{Echantillonnage}

En 1988, un échantillon de 10 arbres a été mesuré en continu de mars à novembre. Les mesures étaient effectuées toutes les $10 \mathrm{~s}$, les moyennes horaires étant calculées et stockées par une centrale d'acquisition Campbell Ltd, 21 $X$.

Ces 10 arbres sont situés à l'intérieur du placeau expérimental; ils ont été choisis de façon à représenter l'ensemble de la gamme des surfaces d'aubier à $1.30 \mathrm{~m}$ du peuplement. Leurs caractéristiques dendrométriques sont données dans le tableau $I$.

En 1987 et en 1989, l'effectif de l'échantillon était respectivement de 7 et 8 individus, choisis de la même façon. Les périodes de mesure se sont prolongées, en 1987 de juillet à septembre, et en 1989 de mai à novembre.

Les sondes ont toutes été insérées dans le tronc à $130 \mathrm{~cm}$ de hauteur dans le secteur azimutal Nord-Est/Sud-Est, abrité des vents dominants.

\section{Estimation de la densité de flux de sève journalière et de la transpiration du peuplement}

Les variations du contenu en eau de la partie aérienne des arbres d'une journée à l'autre ont été négligées et la transpiration journalière du peuplement $\left(\mathrm{mm} \cdot \mathrm{j}^{-1}\right)$ a été assimilée à son débit de sève total. Cette estimation repose sur la relation :

$$
\operatorname{Tr}=J_{s c} \times S A_{c} \times 10^{-4}
$$

Le calcul de cette expression requiert l'estimation de deux paramètres :

- l'estimation de la surface totale d'aubier à l'hectare, $S A_{c}$ a été effectuée par la méthode

Tableau 1. Caractéristiques dendométriques des arbres de l'échantillon mesuré en flux de sève (mesures de 1988).

\begin{tabular}{lccc} 
Numéro Circonférence & Hauteur & $\begin{array}{c}\text { Section } \\
\text { d'aubier à } \\
1.30 \mathrm{~m}\left(\mathrm{dm}^{2}\right)\end{array}$ \\
& à $1.30 \mathrm{~m}(\mathrm{~cm})$ & $(\mathrm{m})$ & \\
\hline 456 & 43 & 11.4 & 0.68 \\
524 & 54 & 11.5 & 1.06 \\
433 & 55 & 12.1 & 1.25 \\
434 & 56 & 11.8 & 1.38 \\
546 & 56 & 12.4 & 1.39 \\
526 & 61 & 12.1 & 1.53 \\
425 & 64 & 12.2 & 1.60 \\
520 & 69 & 13.1 & 1.88 \\
578 & 76 & 13.4 & 2.01 \\
521 & 76 & 13.6 & 2.16 \\
\hline
\end{tabular}


du quotient (Snedecor et Cochran, 1956) : la surface d'aubier de 33 arbres composant un échantilion représentatif de la gamme des circonférences de la parcelle a été estimée par carottage à cœur ( 3 prélèvements par arbre) (EI Hadj Moussa, 1989); une régression linéaire reliant la section d'aubier et la surface terrière individuelles des arbres de cet échantillon a été calculée; la surface totale d'aubier à l'hectare est ensuire calculée à partir de la surface terrière à l'hectare qui a été mesurée sur les 2 ha entourant le placeau.

La régression linéaire calculée entre la section d'aubier et la surface terrière des 33 arbres donne la relation suivante :

$$
S A=0,384 \cdot S T E+32,77(r=0,84 n=33)
$$

L'estimation de la surface d'aubier de la parcelle s'élève en 1988 à :

$$
S A_{c}=1280 \pm 120 \mathrm{dm}^{2} \cdot \mathrm{ha}^{-1}(\alpha=0,05)
$$

- La densité de flux moyenne du peuplement, $J_{s c}$ a tté estimée par la moyenne arithmétique des densités de flux de l'échantillon mesuré.

\section{Mesures de conductance stomatique et de potentiel hydrique}

Les mesures de conductance stomatique, $g_{s}$ ont été effectuées avec un poromètre LI-COR
1600 opérant à humidité constante. La surface foliaire des portions d'aiguilles mesurées a été calculée à partir de leur diamètre en assimilant les aiguilles à un demi cylindre.

En 1988, un échantillon de 20 à 30 paires d'aiguilles de différents àges, réparties sur 5 verticilles supérieurs de l'arbre $n^{\circ} 425$, accessibles depuis un échafaudage, a été mesuré au cours de 10 journées réparties entre mai et novembre (tableau II), a raison d'une journée toutes les 3 semaines environ. Chaque jour, l'échantillon a été entièrement mesuré toutes les heures, entre $9 \mathrm{~h} 00$ et $18 \mathrm{~h} 00 \mathrm{TU}$.

En 1989, l'échantillon a été réduit à 10 paires d'aiguilles réparties sur 3 verticilles et comprenant des aiguilles de l'année et les aiguilles d'un an. Cet échantillon a été mesuré lors de 6 journées entre $9 \mathrm{~h} 00$ et $11 \mathrm{~h} 00 \mathrm{TU}$ (mesures de conductance maximale) et sur deux journées, de 9 h 00 à 18 h 00 TU sur le même arbre.

Ces mesures ont permis d'estimer des moyennes horaires de conductance stomatique pour les différentes journées de mesure; la conductance maximale a été définie comme la moyenne des conductances mesurées avant 11 h 00 TU.

Les mesures de potentiel hydrique ont été effectuées sur le site expérimental, avec une chambre à pression de Scholander. Sur le même arbre, un échantillon de 10 aiguilles $(5$ paires) a été mesuré toutes les heures, lors de chaque journée de mesures. Les cinq paires mesurées étaient réparties sur les cinq verti-

Tableau II. Calendrier des mesures opérées au cours des trois expérimentations. L'effectif de l'échan-

\begin{tabular}{|c|c|c|c|c|}
\hline Année & Flux de Sève & $\begin{array}{l}\text { Cinétiques } \\
\text { journalières de } \\
\text { Gs et Potentiel } \\
\text { hydrique }\end{array}$ & $\begin{array}{l}\text { Mesures du } \\
\text { stock hydrique } \\
\text { du sol }\end{array}$ & $\begin{array}{l}\text { Mesures } \\
\text { météorologiques }\end{array}$ \\
\hline 1988 & $\begin{array}{l}3 / 07 \text { au } 10 / 09 \\
\text { (7) }\end{array}$ & - & - & - \\
\hline 1988 & $\begin{array}{c}10 / 03 \text { au } 11 / 11 \\
(10)\end{array}$ & $\begin{array}{l}14 / 04 \text { 19/05 02/06 } \\
15 / 0608 / 0721 / 07 \\
30 / 0827 / 0726 / 10\end{array}$ & $20 / 07$ au $3 t / 12$ & $01 / 02$ au $31 / 12$ \\
\hline 1989 & $\begin{array}{c}15 / 05 \text { au } 30 / 10 \\
\text { (8) }\end{array}$ & $28 / 0603 / 10$ & $01 / 01$ au $15 / 11$ & $01 / 01$ au $15 / 11$ \\
\hline
\end{tabular}
tillon d'arbres suivis en flux de sève est indiqué entre parenthèses. 
cilles supérieurs et sur le quartier Sud de la couronne. Des mesures de potentiel en fin de nuit ont été opérées lors de quelques journées supplémentaires en 1989. Suivant Aussenac et Granier (1978), le potentiel de base des aiguilles est défini comme le potentiel mesuré en fin de nuit et le potentiel minimum correspond à la valeur la plus basse mesurée au cours de la journée, sur le même matériel.

Le tableau II présente le calendrier des mesures effectuées au cours des trois saisons de mesures $(1987,1988$ et 1989$)$.

\section{Estimation de la conductance hydrauli- que globale racine-feuille}

Si on néglige a priori la quantité d'eau mobilisée dans le courant de sève à partir de réservoirs internes, l'équation de transfert de la sève entre les racines et les aiguilles peut être écrite en utilisant l'analogie électrique (Cohen et al, 1983) :

$$
J_{s}=L_{s} \cdot\left(\psi_{0}-\psi_{\theta}\right)
$$

La conductance hydraulique spécifique, $L_{s}$ et le potentiel à flux nul $\psi_{0}$ ont été estimés par régression linéaire à partir des valeurs de densité de flux et de potentiel hydrique mesurés au cours des journées de mesures complètes (Cohen et al, 1983; Granier et al, 1988). Les hypothèses requises à l'utilisation de cette relation et à l'interprétation des résultats de cette estimation sont discutées plus loin.

\section{Mesures de stock hydrique de sol}

Les mesures décadaires de stock hydrique du sol ont été effectuées par mesure neutronique (humidimètre SOLO 25, CEA, Nardeux) sur un réseau de 8 tubes d'accès neutroniques (atteignant une profondeur de $150 \mathrm{~cm}$ ) et disposés sur les inter-rangs suivant une configuration spatiale optimisée par krigeage (Brejon, 1988; Granier et al, 1990). A partir de la structure spatiale d'une variable sur un domaine donné, définie par son variogramme, le krigeage permet d'interpoler la valeur moyenne de cette variable sur ce domaine et fournit la précision de l'estimation réalisée. Pour définir la configuration du réseau de mesures présentant le meilleur rapport (précision/nombre de points de mesure), différents réseaux ont été comparés à partir d'un jeu de données expérimentales collecté sur ce domaine. Cette procédure est décrite plus en détail par Brejon (1988) et Granier et al (1990).

Entre chaque journée de mesure, les valeurs de stock ont été estimées à partir de la mesure de stock la plus proche dans le temps et d'une estimation des variations de stock opérée à partir des mesures de flux de sève des arbres, de I'ETR du sous-étage de Molinie (Cochard, 1988; Granier et al, 1990) et des flux de remontées capillaires.

La profondeur maximale d'enracinement observée dans la parcelle est de $70 \mathrm{~cm}$; la profondeur maximale de sol utile a été estimée à 75 $\mathrm{cm}$.

La réserve utile du sol a été estimée entre 100 et $110 \mathrm{~mm}$ à partir de mesures du stock maximal au printemps, sur sol ressuyé $\left(R_{c c}=\right.$ $140 \mathrm{~mm}$ ), et minimal, sur sol sec au milieu de l'été 1989, année exceptionnellement sèche $\left(R_{m}\right.$ $=31 \mathrm{~mm}$ ).

Cette estimation permet d'exprimer les stocks mesurés en pourcentage d'eau extractible par la végétation (PEE):

$$
P E E=\frac{R-R_{m}}{R_{c c}-R_{m}} \times 100
$$

\section{Mesures micrometéorologiques}

Les moyennes horaires de température, humidité relative, vitesse et direction du vent, rayonnement global, pluviométrie sont calculées à partir de mesures opérées toutes les $20 \mathrm{~s}$ par une station météorologique automatique Campbell située à $15 \mathrm{~m}$ au dessus du sol au centre du placeau expérimental.

A partir de ces donnés, une valeur d'évapotranspiration potentielle horaire a été calculée avec la relation de Penman, adaptée à partir de données plus récentes sur la résistance aérodynamique des couverts de résineux:

$$
E T P=\frac{s \cdot(A)+g_{a} \cdot p \cdot C_{p} \cdot D S A T}{\lambda \cdot(s+\eta} \times 3600
$$


Le terme $A$, énergie disponible, a été estimé empiriquement, à partir des mesures de rayonnement global, $R g$, par :

$$
A=0,75 R g-0,15
$$

La conductance aérodynamique, $g_{a}$ a été calculée en utilisant la relation de Monteith (1973) et les estimations de Jarvis et al (1976) de hauteur de plan de référence (d) et de rugosité $\left(z_{o}\right)$ pour les couverts de conifères :

$z_{0}=0,075 h ; d=0,78 h(h$ : hauteur moyenne du couvert $(=13 \mathrm{~m})$ )

$$
g_{a}=\frac{k^{2} \cdot U_{z}}{\left[\operatorname{Ln}\left(\frac{z-d}{z_{0}}\right)\right]}
$$

En 1987, ces mesures n'ont pas été déterminées sur le site, et les données d'évapotranspiration (ETP) utilisées ont été celles de la station de Mérignac, distante d'une dizaine de $\mathrm{km}$ à vol d'oiseau du site expérimental. En revanche, les données pluviométriques de cette station n'ont pas été transposées, la variabilité spatiale des champs pluviométriques n'étant pas connue.

\section{RÉSULTATS}

\section{Variabilité de la densité de flux de sève}

Quelles que soit l'année d'expérimentation considérée, la densité de flux de sève d'un arbre ne semble pas pouvoir être reliée de façon simple à l'une de ses caractéristiques dimensionnelles (circontérence, hauteur, section d'aubier à $1,30 \mathrm{~m}$, surface foliaire).

La figure 1 présente la relation obtenue pour quelques journées de chaque expérimentation, entre la densité de flux de sève (cumul journalier) et la section d'aubier de chaque arbre mesuré.

La variabilité de la densité de flux de sève est plus élevée en condition de flux élevé et diminue quand le flux de sève est plus fáible.

Cette absence d'une relation marquée entre densité de flux de sève et dimension d'un arbre se retrouve quelle que soit l'échelle de temps (heure, jour, décade) ou la période considérée.

Le coefficient de variation des mesures de densité de flux varie entre 20 et $30 \%$ au cours des 3 années de mesure et permet une précision de $\pm 15 \%$ sur l'estimation de la densité de flux de sève moyenne à l'hectare.

L'estimation de la densité de flux de sève moyenne du peuplement a été faite avec la moyenne arithmétique des densités de flux de l'échantillon.

\section{Evolution saisonnière de la densité de flux et de la transpiration du peuplo- ment}

En 1988, la densité de flux de sève moyenne du peuplement augmente graduellement de mi-mai à fin-juin; elle présente en juillet et août des valeurs maxi-

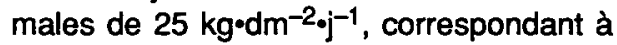
une transpiration journalière de 3 à 3,5

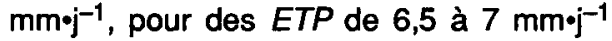
(fig 2). Jusqu'au mois de septembre, les variations de débit de sève sont globalement parallèles aux fluctuations de I'ETP journalière. Durant cette période, le stock hydrique du sol est toujours supérieur à $140 \mathrm{~mm}$; il diminue rapidement à partir du mois d'août et atteint des valeurs comprises entre 40 et $50 \mathrm{~mm}$ en septembre.

Au milieu du mois de septembre, la densité moyenne de flux de sève est réduite brutalement à des valeurs inférieures à 8 $\mathrm{kg} \cdot \mathrm{dm}^{-2} \cdot \mathrm{j}^{-1}$, correspondant à une transpiration de $1 \mathrm{~mm} \cdot \mathrm{j}^{-1}$. Pendant la fin du mois de septembre, les variations journalières de transpiration sont très atténuées relati- 

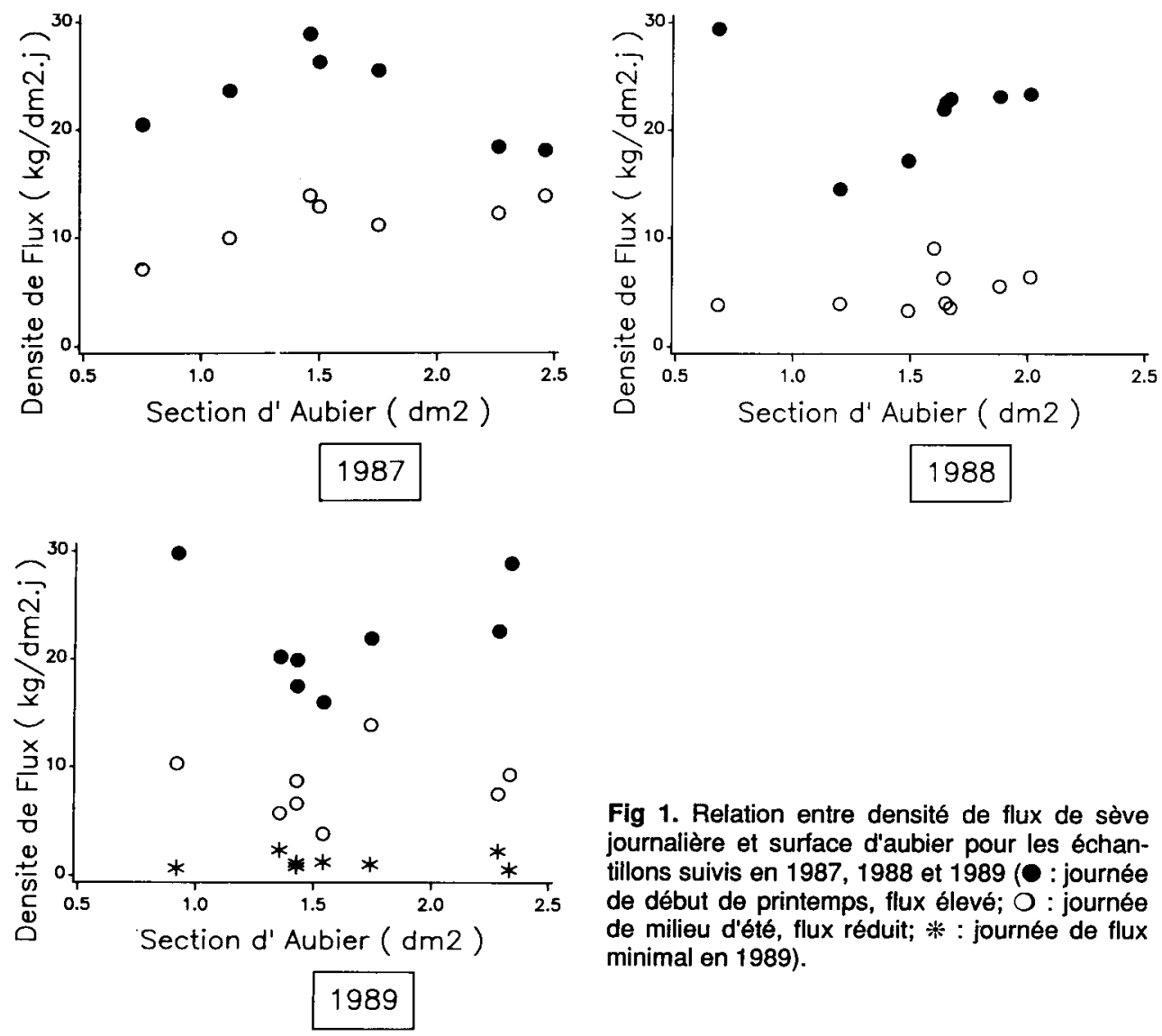

vement à celles de I'ETP; en octobre, le stock hydrique remonte entre 50 et $60 \mathrm{~mm}$ à la suite d'une série d'épisodes pluvieux. II a fluctué entre ces deux valeurs jusqu'à la fin de l'expérimentation. Une remontée du débit de sève consécutive aux épisodes pluvieux et à cette augmentation du stock hydrique est observée durant cette période.

En 1987 et 1989, l'évolution saisonnière du débit de sève et de la transpiration du peuplement diffère essentiellement par la durée et par la date de la réduction de la transpiration. En 1987, cette réduction est survenue vers le milieu du mois d'août, et s'est prolongée jusqu'à l'arrêt des me-

sures, à la mi-septembre. Au cours de l'année 1989, particulièrement déficitaire en précipitations, cette réduction a eu lieu dès la fin du mois de juin et s'est prolongée au delà de la fin octobre. Cette chute de débit de sève a atteint une ampleur particulière à trois reprises en 1989 : durant quelques jours précédant les pluies de la fin juillet, entre le $1^{\text {er }}$ et le 15 septembre et vers la mi-octobre. Le flux de sève indivi-

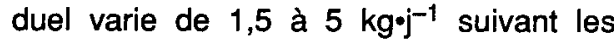
arbres et la transpiration journalière atteint des minima de 0,12 à $0,18 \mathrm{~mm} \cdot \mathrm{j}^{-1}$ (31 août-15 septembre). Ces trois périodes coïncident avec des stocks hydriques du sol inférieur à $35 \mathrm{~mm}$ (PEE de $5 \%$ ). 
Ces réductions de flux de sève sont enregistrées pour tous les arbres mesurés dans le même laps de temps de 2 à 3 jours, ce qui traduit une relative simultanéité de la réduction du flux transpiratoire pour les différents arbres composant les échantillons suivis au cours des trois campagnes de mesure.

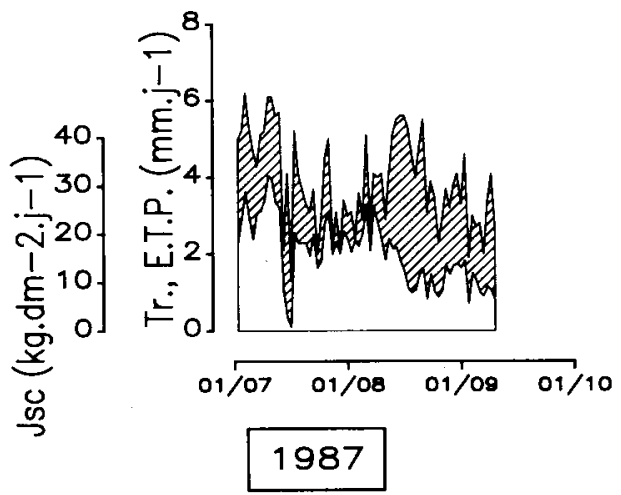

En 1988, comme en 1989, la réduction du flux de sève s'observe quand le stock hydrique du sol $(0-75 \mathrm{~cm})$ atteint un seuil situé entre 50 et $55 \mathrm{~mm}$, correspondant à un PEE de 15 à $20 \%$. En deçà de ce seuil, les variations journalières de débit de sève ne suivent plus celles de l'ETP mais répondent surtout a l'occurence des épisodes pluvieux qui sont suivis de remontées de

Fig 2. Évolutions saisonnières de la densité moyenne de flux (ou transpiration), de l'ETP et de la pluviométrie journalière du peuplement de pins. L'aire hachurée oblique délimite la différence entre l'ETP (courbe supérieure) et la densité moyenne de flux du peuplement de pins (courbe inferieure). Le diagramme en bâtonnets représente la hauteur journalière des précipitations. La figure inférieure représente l'évolution du stock hydrique $(0-75 \mathrm{~cm})$ du sol (0).
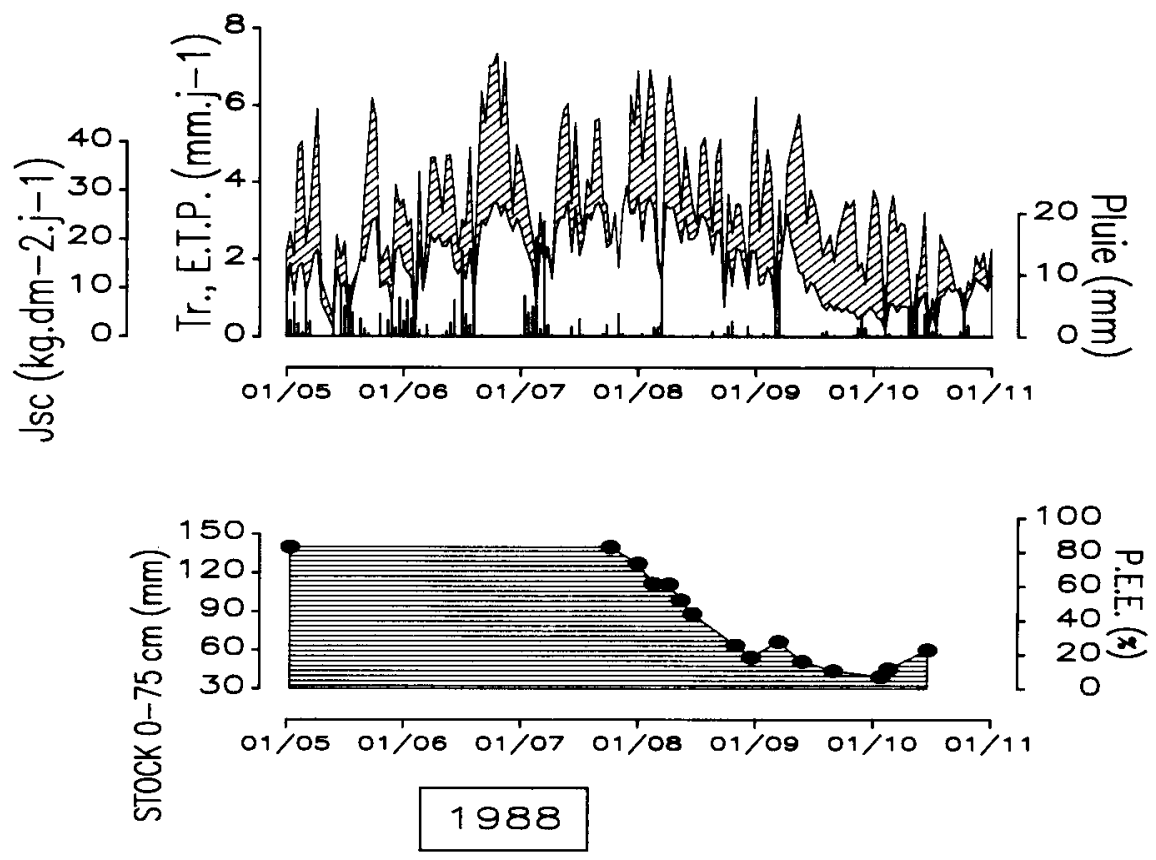

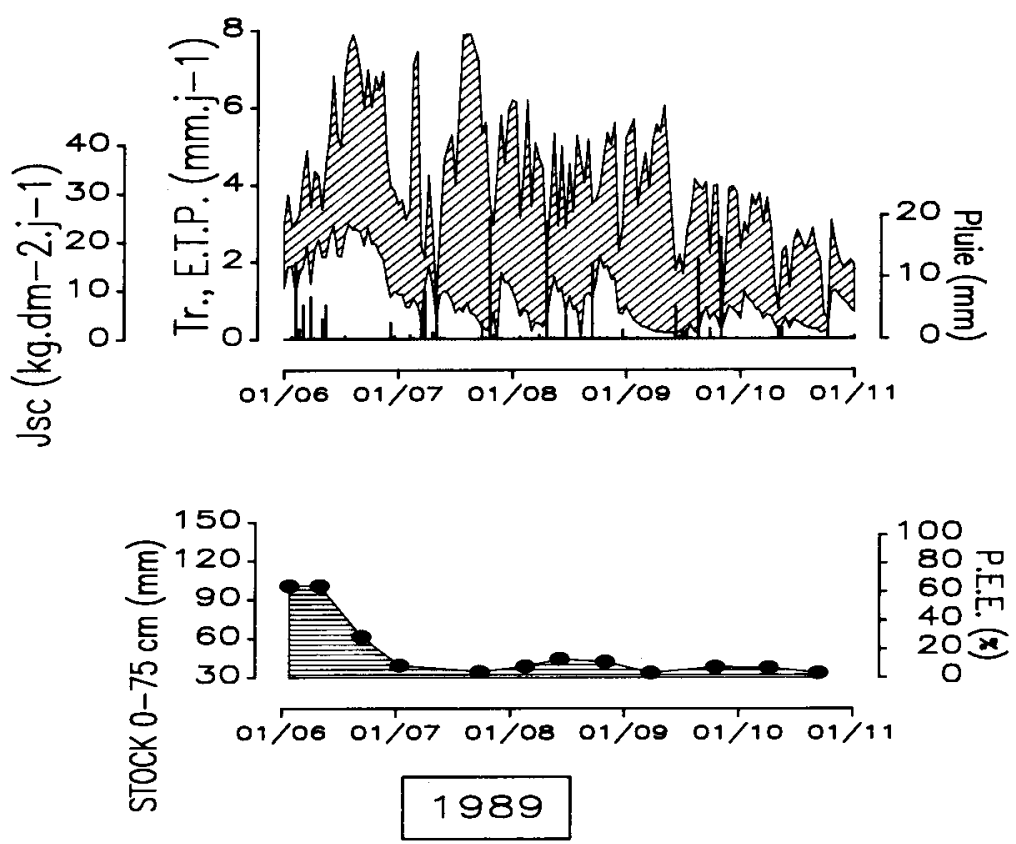

flux de sève. Les valeurs de densité de flux atteintes restent cependant inférieures aux valeurs mesurées sur sol bien pourvu en eau.

La relation entre $P E E$, potentiel de base et potentiel minimum est illustrée par la figure 3. Pour des PEE supérieurs à $25 \%$ (stock supérieur à $60 \mathrm{~mm}$ ), les valeurs de potentiel de base varient entre $-0.3 \mathrm{MPa}$ et $-0.5 \mathrm{MPa}$; en deçà de ce seuil, le potentiel de base diminue rapidement avec le dessèchement du sol, jusqu'à un minimum de $-1.16 \mathrm{MPa}$, mesuré pour un PEE de 1 à $2 \%$, équivalent à un stock de $32 \mathrm{~mm}$, à la fin du mois d'août 1989. En revanche, le potentiel minimal des aiguilles varie relativement peu; il fluctue autour d'une valeur de $-1.5 \mathrm{MPa}$, et semble globalement indépendant des conditions d'humectation du sol.

La figure 4 montre la relation entre la conductance stomatique maximale et le potentiel de base, établie à partir des données mesurées en 1988 et 1989.
Malgré la dispersion des valeurs de conductance mesurées le matin, la diminution de conductance quand le potentiel de base diminue est manifeste; elle semble constante tout au long de la gamme de potentiel observée. L'équation de la droite de régression (proc REG logiciel SAS) entre la conductance maximale (en $\mathrm{cm}^{-1} \mathrm{~s}^{-1}$ ) et le potentiel de base (en MPa) peut s'écrire : $g_{s}=0,56 \cdot \psi_{b}+0,49\left(n=182 ; r^{2}=0,42 ; \alpha=\right.$ 0,001 )

\section{Cinétiques journalière des relations hy- driques}

Parmi les 12 journées de mesure réalisées au cours des expérimentations de 1988 et 1989, deux types d'évolution journalière de conductance stomatique, potentiel hydrique, et flux de sève se différencient nettement (fig 5).

Au printemps et au début de l'été (fig 5a), quand le PEE est supérieur à $20 \%$, la 
conductance stomatique présente des valeurs décroissantes du matin au soir, de 0,30 a $0,10 \mathrm{~cm} \cdot \mathrm{s}^{-1}$; le potentiel hydrique présente des valeurs maximales de $-0,3$ MPa en fin de nuit et atteint des valeurs minimales de $-1,5 \mathrm{MPa}$ en milieu de journée.

Le flux de sève horaire varie de façon parallèle avec I'ETP et présente une augmentation rapide le matin, une phase stationnaire en milieu de journée et une décroissance plus graduelle dans l'aprèsmidi. II tend à s'annuler vers minuit. Juste avant $16 \mathrm{~h} 00$, un passage nuageux a provoqué une baisse de I'ETP horaire qui coïncide avec une chute du flux de sève et une remontée de potentiel hydrique foliaire.

A partir de la fin de l'été (fig $5 b$ ), pour des PEE inférieurs à $20 \%$, le potentiel hydrique en fin de nuit diminue et atteint $-0,7$ pour la journée représentée; en milieu de journée il reste cependant compris entre 1,3 et $-1,6 \mathrm{MPa}$.

La conductance stomatique présente des valeurs matinales plus faibles que précédemment, de 0,10 à $0,20 \mathrm{~cm} \cdot \mathrm{s}^{-1}$, et se réduit précocement à des valeurs d'environ $0,05 \mathrm{~cm} \cdot \mathrm{s}^{-1}$. La conductance stomatique des jeunes aiguilles reste cependant élevée, relativement à celle des aiguilles d'un an.

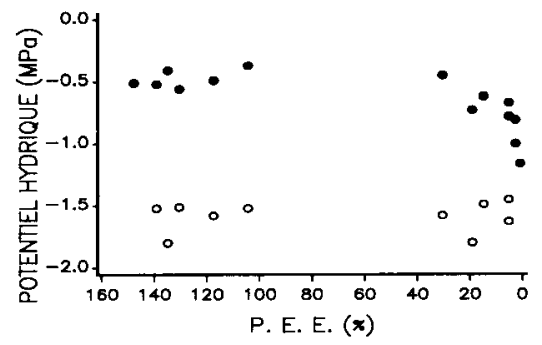

Fig 3. Relation entre potentiel de base (O), potentiel minimum (O) et pourcentage d'eau extractible (mesures de 1988 et 1989).
Les valeurs horaires de densité de flux de sève sont réduites d'un facteur 3 à 4 par rapport aux valeurs atteintes précédemment. Elles restent comprises entre 0,5 et $0,7 \mathrm{~kg} \cdot \mathrm{dm}^{-2} \cdot \mathrm{h}^{-1}$ sur la plus grande partie de la journée.

\section{Estimation de la conductance globale spécifique racines-feuilles}

Le tableau III présente les valeurs de $y_{0}$ et de $L_{s}$ pour différentes journées de mesure caractéristiques. Les diagrammes fluxpotentiels de six de ces journées, présentant des niveaux variés de réserve hydrique du sol, sont représentés dàns la figure 6.

Au cours du dessèchement du sol, deux phénomènes se manifestent : une diminution du potentiel à flux nul $\psi_{0}$ et une réduction de la conductance globale au transfert de sève dans l'arbre. Une bonne corrélation est observée entre potentiel de base et potentiel à flux nul (fig 7 ). La réduction de la conductance hydraulique globale se traduit par la réduction de la pente des droites de régression flux-potentiel quand le PEE diminue. La figure 8 montre l'évolution de la conductance hydraulique globale quand le potentiel de base diminue.

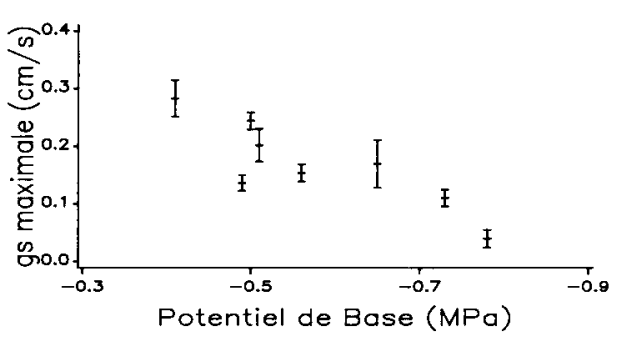

Fig 4. Relation entre conductance stomatique maximale $\left(g_{\mathrm{s}}\right)$ et potentiel de base. Les barres verticales représentent l'erreur-standard sur $g_{\mathrm{s}}$. 

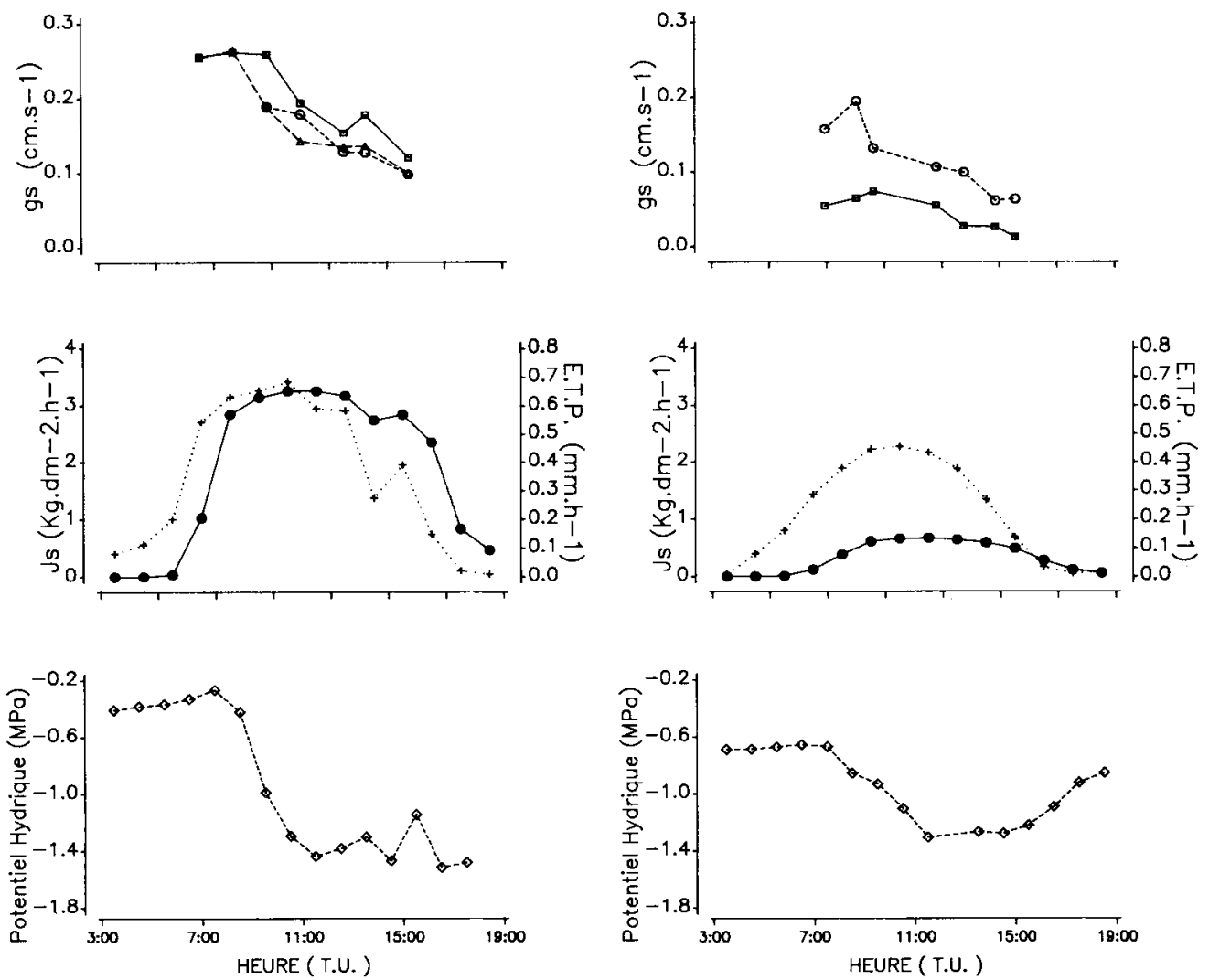

A

$B$

Fig 5. Cinétiques journalières de conductance stomatique classée par âge des aiguilles $(O$ : aiguille de l'année; $\square$ : aiguille d'un an; $\Delta$ : aiguille de deux ans), flux de sève (๑), ETP (+) et potentiel hydrique foliaire $(0)$ pour deux journées de mesure. A: sol bien pourvu en eau, PEE $=120 \%$; $B$ : sol en phase d'assèchement, PEE $=5 \%$.

\section{DISCUSSION}

\section{Variabilité interarbres de la densité de flux de sève}

A l'échelle du peuplement, la variabilité des mesures de densité de flux de sève intègre deux composantes : une compo- sante intra-arbre (variabilité azimutale, radiale, suivant la hauteur de la sonde sur le tronc...) et une composante inter-arbre. Le protocole d'échantillonnage a été conçu de façon à réduire au minimum les effets de variabilité intra-arbre. Celle-ci peut cependant être importante comme cela a été montré chez le Douglas (Cohen et al, 1984). 
Tableau III. Niveau de la réserve hydrique du sol, du potentiel de base, potentiel à flux nul $\psi_{0}$, et conductance spécifique globale $L_{s}$ calculés pour six journées de mesure (arbre numéro 425).

\begin{tabular}{|c|c|c|c|c|c|c|}
\hline \multirow{2}{*}{$\begin{array}{l}\text { Stock } \\
0-75 \mathrm{~cm} \\
(\mathrm{~mm})\end{array}$} & \multirow{2}{*}{$\begin{array}{l}\text { PEE } \\
\text { (\%) }\end{array}$} & \multirow{2}{*}{$\begin{array}{l}\text { Potentiel } \\
\text { de base } \\
\text { (MPa) }\end{array}$} & \multicolumn{3}{|c|}{$\begin{array}{l}\text { Paramètres de la régression } \\
\text { potentiel/densité de flux }\end{array}$} & \multirow[b]{2}{*}{$N$} \\
\hline & & & $\begin{array}{l}\text { Potentiel a } \\
\text { flux nul } \\
(M P a)\end{array}$ & $\begin{array}{c}\text { Conductance } \\
\text { specifique globale } \\
\left(\mathrm{kg} \cdot \mathrm{dm}^{-2} \cdot \mathrm{h}^{-1} \cdot \mathrm{MPa}\right)\end{array}$ & $r^{2}$ & \\
\hline 200 & 150 & -0.56 & -0.66 & 4.16 & 0.92 & 15 \\
\hline 190 & 140 & -0.41 & -0.41 & 2.97 & 0.86 & 15 \\
\hline 155 & 110 & -0.49 & -0.50 & 3.43 & 0.95 & 14 \\
\hline 65 & 22 & -0.45 & -0.56 & 3.58 & 0.91 & 14 \\
\hline 48 & 15 & -0.62 & -0.63 & 1.72 & 0.94 & 20 \\
\hline 40 & 08 & -0.78 & -0.84 & 1.26 & 0.98 & 11 \\
\hline 36 & 05 & -0.68 & -0.74 & 1.21 & 0.93 & 19 \\
\hline
\end{tabular}

L'absence de relation simple entre la densité de flux de sève et la dimension des arbres suggère qu'une telle relation, si elle existe, ne rend compte que d'une faible part de la variabilité des mesures dans nos conditions expérimentales. Ceci peut s'expliquer à la fois par une prédominance de la variation intra-arbre et par la physionomie du peuplement étudié :

- le placeau expérimental présente une forte régularité d'espacement, de topographie et de végétation de sous-étage et les statuts concurrentiels sont relativement peu contrastés.

- la canopée présente un aspect très ouvert, typique des couverts de pin maritime, qui atténue les variations de conditions environnementales entre les houppiers; les houppiers des arbres de faible classe de circonférence et hauteur atteignent l'enveloppe supérieure de la canopée.

Cette relative homogénéité est observée chez le pin ponderosa (Lopushinsky, 1986), et par certains auteurs (Kline et al, 1976) dans des peuplements de Douglas. D'autres auteurs trouvent au contraire une relation croissante entre la densité de flux de sève d'un arbre et son statut concurrentiel (Lopushinsky, 1986; Granier, 1987). Dans un peuplement de pins maritimes de 37 ans récemment éclairci, Granier et al (1989) observent une relation faiblement croissante entre densité de flux et circonférence des arbres. Les conditions expérimentales différentes rendent cependant toute comparaison impossible.

\section{Variations saisonnières du flux de sève et de la transpiration}

Diawara et al (1990), ont montré à partir de mesures effectuées sur ce même site en 1988, que les estimations de transpiration journalière opérées à partir du flux de sève concordent à $15 \%$ près avec celles opérées à partir du bilan d'énergie du couvert. Cet écart rentre dans l'intervalle de confiance des différentes estimations.

Au cours des trois expérimentations, les variations de la densité de flux de sève journalière correspondent à deux types de facteurs : quand le PEE est supérieur à $20 \%$ elles répondent aux variations $d^{\prime} E T P$ 

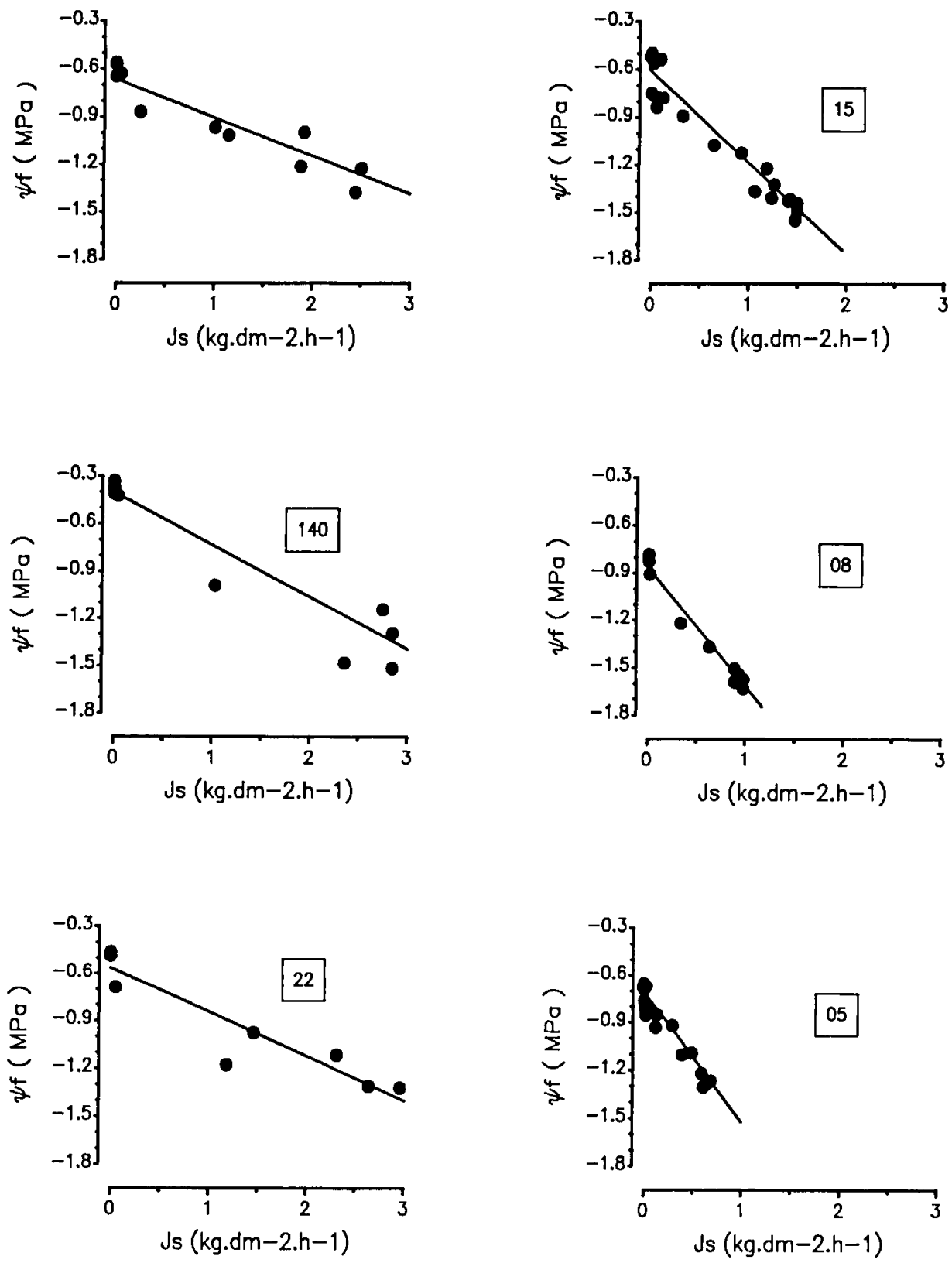

Fig 6. Relation entre densité de flux et potentiel hydrique pour 6 journées de mesure classées par ordre de contrainte hydrique croissante. Le PEE correspondant à chaque journée est encadré. 


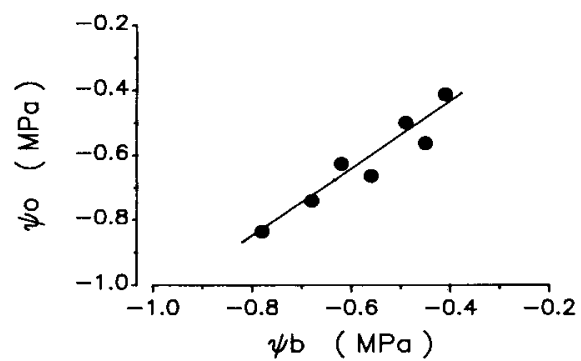

Fig 7. Relation entre le potentiel hydrique à flux nul $\left(\psi_{0}\right)$ et le potentiel de base. (L'équation de la droite de régression est : $\psi_{0}=1,03 \times \psi_{b}-0,03$; $r^{2}=0,90 ; n=7 ; \alpha=0.001$ ).

journalières; en deçà, elles semblent surtout liées au niveau hydrique du sol.

La densité de flux de sève s'exprime comme le produit de deux termes : la différence de potentiel hydrique entre le sol et la feuille d'une part et la conductance hydraulique globale entre ces deux points d'autre part.

$$
J_{s}=\left(\psi_{t}-\psi_{s t}\right) \times L_{s}
$$

Cette expression implique un certain nombre d'hypothèses discutées par exemple chez Passioura (1984) : pas de flux de solutés dans le système, le système est isotherme, la conductance hydraulique est indépendante des variations du potentiel hydrique dans la plante ou de ses composantes. Ces hypothèses sont peu restrictices dans le cas d'un végétal de grande dimension en conditions naturelles, et la simplicité de cette formulation permet une analyse appropriée à l'étude d'une plante entière (Katerji et al, 1982; Cohen et al, 1983; Cruiziat et Granier, 1988). Le flux de sève est donc décrit et analysé ici dans son expression la plus simple, suivant un circuit global sol-feuille.

Le passage de l'expression (1) à l'expression (2), utilisée dans le calcul $L_{s}$ et de

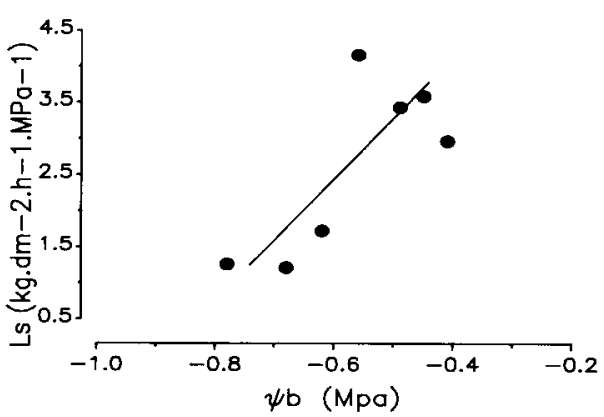

Fig 8. Evolution de la conductance hydraulique globale, $L_{s}$, suivant le potentiel de base. La droite ajustée à la main souligne simplement la tendance générale.

$\psi_{0}$, suppose que le potentiel à l'interface sol-racine corresponde à $\psi_{0}$. La figure 7 montre que le potentiel $\psi_{0}$ correspond globalement au potentiel de base, qui traduit le potentiel hydrique à l'interface racinesol, $\psi_{s r}$ (Aussenac et Granier, 1978). A cet égard, la différence de potentiel ( $\psi_{r}$ $\psi_{0}$ ) peut donc bien être assimilé au gradient de potentiel sol-feuille.

Par ailleurs, les expressions (1) et (2) supposent que le flux doit être conservatif le long du circuit sol-feuille. Cette condition n'est en fait jamais vérifiée chez un végétal. La plupart des modèles de fonctionnement hydrique des végétaux introduisent différents réservoirs hydriques dans la plante (Katerji et al, 1982; Cruiziat et Granier, 1988; Tyree, 1988). Cependant, la dispersion des points des diagrammes flux-potentiel autour de la droite de régression (fig 6) suggère que la quantité d'eau journellement stockée et déstockée dans les parties aériennes de l'arbre est relativement faible par rapport au flux transpiratoire journalier et que les conditions de flux conservatif sont réalisées en première approximation.

Selon l'état des réserves hydriques du $\mathrm{sol}$, on peut distinguer principalement deux types de comportement hydrique. 
- Quand le pourcentage d'eau extractible est supérieur à $20 \%$, les variations journalières de densité de flux suivent étroitement celles de l'ETP (fig 2). A l'échelle horaire, les variations de densité de flux correspondent à des variations de potentiel hydrique foliaire, comme le montrent les cinétiques journalières de flux et de potentiel hydrique (fig 5). La capacité en eau du sol peut être considérée comme infinie et le potentiel hydrique à l'interface sol-racine peut être supposé constant au cours de la journée. Les variations horaires de la différence $\left(\psi_{f}-\psi_{0}\right)$ correspondent aux seules variations de potentiel hydrique foliaire. Durant cette période, les valeurs mesurées de potentiel de base $(-0.4$ à $-0.56 \mathrm{MPa}$, tableau III) correspondent à la gamme de valeurs observées pour des espèces du genre Pinus (Hellkvist et Parsby, 1976; Running, 1980; Aussenac et Valette, 1982). Ces valeurs sont notablement inférieures à celles du potentiel hydrique du sol qui sont proches de 0 MPa durant cette période. Les causes de ces écarts entre potentiel en fin de nuit et potentiel hydrique du sol ne sont pas établies lors de journées à forte ETP, ils pourraient être en partie attribués à une réhydratation incomplète de l'arbre. Selon l'équation (1), les fluctuations de densité de flux de sève journalières sur sol bien pourvu en eau correspondent aux variations du cumul journalier des différences de potentiels $\left(\psi_{f}-\psi_{0}\right)$.

- La diminution du PEE en deçà de $20 \%$, s'accompagne en premier lieu d'une diminution du potentiel de base, qui atteint des valeurs allant de -0.6 à $-1.2 \mathrm{MPa}$ (fig 3). La valeur minimale de potentiel de base de $-1.2 \mathrm{MPa}$, mesurée au cours de l'été 1989 ne représente qu'une limite supérieure de potentiel de base minimum possible, ces mesures n'étant effectuées que tous les 10 jours. II est vraisemblable que le potentiel de base ait atteint des va- leurs inférieures à $-1.2 \mathrm{MPa}$. Aussenac et Valette (1982) ont mesuré des minima de potentiel de base de $-1.7 \mathrm{MPa}$ en zone méditerranéenne sur cette essence. La valeur de $-1.2 \mathrm{MPa}$ est par ailleurs élevée comparativement aux potentiels de base minimaux atteints par d'autres espèces de conifères en phase de sécheresse, en conditions naturelles : $-1.6 \mathrm{MPa}$ chez le Douglas, $-2.0 \mathrm{MPa}$ chez Pinus ponderosa (Lopushinsky, 1986) ou -1.9 MPa chez Pinus contorta (Fetcher, 1976). Le seuil de $20 \%$ du PEE auquel est observée cette réduction de flux de sève, ainsi que l'allure générale de la courbe potentiel de basePEE, sont comparables aux résultats mentionnés par Running et al (1975) chez Pinus contorta.

\section{Relations hydriques}

Quand le PEE est inférieur à $20 \%$, le pin maritime tend à maintenir un potentiel minimal stable (fig 3). Ce maintien peut être considéré comme le résultat du contrôle stomatique du flux transpiratoire. Chez les conifères, le flux transpiratoire est en effet étroitement dépendant de la conductance stomatique, celle-ci étant d'un à deux ordres de grandeur inférieure à la conductance aérodynamique du couvert (Jarvis et Mc Naughton, 1985). La baisse de conductance stomatique fréquemment observée entre $12 \mathrm{~h} 00$ et $14 \mathrm{~h} 00$ (TU) lors de journées à forte ETP et sur sol bien pourvu en eau, correspond à un potentiel foliaire de $1.5 \mathrm{MPa}$ et pourrait répondre à ce comportement. Cette réduction de conductance stomatique s'interprète généralement par le jeu d'interactions entre facteurs internes (état hydrique de la plante...) et externes (déficit de saturation en vapeur d'eau de l'air) (Schulze et al, 1987) dont la nature même est encore imparfaitement comprise. 
Au cours du développement des épisodes de sécheresse édaphique, ce contrôle s'exprime par une diminution des valeurs de conductance stomatique maximale quand le potentiel de base diminue (fig 4), et, dans une moindre mesure, par une chute de conductance stomatique plus précoce dans la journée (fig 5). Les valeurs de $g_{s}$ maximales mesurées avant la phase de stress sont comparables à celles de la plupart des conifères, qui se situent entre 0,2 et $0,3 \mathrm{~cm} / \mathrm{s}$ (Hinckley et al, 1978). Dès que le potentiel de base diminue en deçà de $-0.6 \mathrm{MPa}$, on observe une réduction de la conductance stomatique maximale qui est divisée par un facteur 5 à 6 . La différence de conductance constatée entre aiguilles de l'année et aiguilles d'un et deux ans suggère que celles-ci transpirent moins. Cette réduction pourrait diminuer l'efficacité de la thermorégulation des aiguilles âgées et les exposer à des dégradations métaboliques plus importantes expliquant en partie l'accélération de la sénescence et de la chute des aiguilles observées au cours des phases de déficit hydrique.

Le maintien d'un potentiel minimal stable, alors que le potentiel de base diminue, entraîne une réduction du gradient de potentiel développé entre le sol et la feuille; ce gradient peut atteindre 1.0 MPa en milieu de journée, sur sol humide, contre 0.6 à $0.5 \mathrm{MPa}$ sur sol sec (fig 3). Cette réduction entraîne virtuellement une chute de la densité de flux de sève de $\mathbf{4 0}$ à $50 \%$ par rapport à sa valeur précédente.

\section{Variation de conductance hydrique}

Parallèlement à ces modifications du gradient de potentiel sol-feuille, le déficit hydrique édaphique induit une modification des caractéristiques hydrauliques du transfert de sève. Les valeurs de conductance spé- cifique $\left(L_{s}\right)$ calculées (tableau III) sont comparables à celles données par Granier et al (1988) pour d'autres résineux. La réduction de $L_{s}$ observée en phase de déficit hydrique est rapportée chez d'autres espèces du genre Pinus, $P$ sylvestris (Pena et Grace, 1986), $P$ contorta (Running, 1980) et d'autres conifères (Abies bornmulleriana, Picea abies (Granier et al, 1988). Cette réduction est ici observée dés que le potentiel de base diminue en deçà de $-0,6$ $\mathrm{MPa}$ et peut aller jusqu'à réduire de deux tiers la capacité conductrice globale de l'arbre entre racines et feuilles (tableau III; fig 8). Cette réduction semble indépendante des valeurs de potentiel foliaire minimales qui varient peu d'une journée à l'autre, même en cas de stress et peut s'interpréter par des modifications des capacités de transfert hydrique à deux niveaux.

- A l'interface sol-racine, le potentiel hydrique peut accuser une importante diminution en milieu de journée. Cette diminution s'explique par un déséquilibre entre le prélèvement racinaire et la mobilisation de l'eau du sol autour de la racine. Sur sol sec, la conductivité hydraulique réduite du sol ne permettrait plus un apport d'eau suffisant pour compenser le prélèvement racinaire en milieu de journée; ce déséquilibre induit une déshydratation passagère de linterface sol-racine et une diminution du potentiel hydrique du sol en cours de journée. Cohen et al (1983) interprètent de cette façon l'accroissement des écarts entre $\psi_{0}$ et le potentiel de base quand le sol de dessèche; mais cette tendance ne se manifeste pas clairement ici (fig 7). Sous cette hypothèse, le calcul de $L_{s}$ par régression linéaire à partir de valeurs horaires de flux et de potentiel est artificiel et ne traduit plus la conductance hydraulique racines-feuilles. Les variations de celle-ci ne sont plus accessibles par ce calcul.

- La conductance spécifique de certains tissus de transfert de l'arbre peut varier 
suivant leur teneur en eau (Edwards et al, 1986) ou être modifiée par l'occurrence de phénomènes comme la cavitation de trachéides du xylème, au cours d'un stress hydrique (Dixon et al, 1984; Tyree et Dixon, 1986; Pena et Grace, 1986). L'importance de tels phénomènes en conditions naturelles n'est cependant pas établie. La cavitation de trachéides du xylème s'observe en réponse à une chute du potentiel hydrique de la sève qui peut elle même être due à une chute du potentiel racinaire. Les modèles récents d'architecture hydraulique des arbres (Tyree, 1988) n'interdisent pas de supposer qu'une diminution du potentiel de sève susceptible de déclencher des phénomènes de cavitations dans certaines parties de l'arbre soit induite par une diminution du potentiel racinaire. Cette chute de potentiel ne se répercute pas nécessairement sur le potentiel hydrique foliaire qui dépend essentiellement de la régulation du flux transpiratoire. II est donc possible que les capacités de transfert de sève interne à l'arbre soient altérées en réponse à une chute du potentiel de base sans que des valeurs minimales de potentiel hydrique foliaire soient modifiées.

\section{CONCLUSION}

A travers cette expérimentation, le comportement hydrique du pin maritime se caractérise par le maintien d'un état hydrique foliaire stable au cours de phases de sécheresse édaphique prolongées. Ce maintien est dû à une limitation stomatique précoce des pertes en eau. La réduction simultanée du flux de sève répond à une diminution du gradient de potentiel hydrique sol-feuilles et de la conductance hydraulique globale du circuit sol-feuilles. L'appréhension des phénomènes de transfert hydrique à l'interface sol-racines apparaît comme une étape nécessaire pour aller plus loin dans l'analyse du fonctionnement hydrique de l'arbre. Elle pourrait permettre de distinguer les mécanismes de transfert entre le sol et la plante de ceux internes à l'arbre.

Les effets des épisodes de sécheresse édaphique les plus graves sur ces deux types de mécanismes pourraient se répercuter sur le fonctionnement hydrique de cette essence à des échelles de temps allant jusqu'à l'année, par exemple par l'induction de déséquilibres entre capacité conductrice de sève et surface transpirante de l'arbre.

L'observation de stress hydriques au cours de chacune de ces trois saisons climatiquement contrastées, dans un site localement considéré comme humide, confirme la nécessité d'une prise en compte du fonctionnement hydrique du pin maritime dans la conduite des peuplements landais. Le développement de ce programme par des expérimentations destinées à évaluer la consommation en eau de la strate herbacée et les effets d'une éclaircie sur le bilan hydrique d'un peuplement devrait donner certains éléments de réponse à cet objectif.

\section{REMERCIEMENTS}

Une partie des données utilisées dans cet article sont issues des stages de $M^{\text {lle }}$ Ducasse (ENITHP, Angers) et de M Brejon de la Vergnée (ENSA, Rennes). La dactylographie de cet article a été effectuée par $M^{m \theta}$ Guillem. Les auteurs les en remercient. Durant son travail de thèse au Laboratoire d'écophysiologie de l'INRA à Cestas, F El Hadj Moussa a été soutenu par une bourse du gouvernement algérien.

\section{RÉFÉRENCES}

Aussenac G, Granier A (1978) Quelques résultats de cinétique journalière de potentiel de 
sève chez les arbres forestiers. Ann Sci For 35, 19-32

Aussenac G, Valette (1982) Comportement hydrique estival de Cedrus atlantica Manetti, Quercus ilex $L$ et Quercus pubescens Wild et de divers pins dans le Mont Ventoux. Ann Sci For 39, 41-63

Aussenac G, Granier A, Naud R (1982) Influence d'une éclaircie sur la croissance et le bilan hydrique d'un jeune peuplement de Douglas Pseudotsuga Menziesii. Can J For Res 12, 222-231

Baradat P, Marpeau-Bezard A (1988) Le pin maritime Pinus pinaster Ait. Biologie et génétique des terpènes pour la connaissance et l'amélioration de l'espèce. Thèse, Université Bordeaux I

Brejon de la Vergnée G (1988) Optimisation d'un réseau de mesures neutroniques à l'aide de la théorie des variables régionaliées. DEA, ENSA Rennes

Choisnel E, Payen D, Lamarque P (1987) Climatologie de la zone du projet HAPEXMOBILHY. Direction de la Météorologie Nationale, Paris, 1987, $75 p$

Cochard $H$ (1988) Utilisation d'un système de type enceinte fermée pour l'estimation de la transpiration journalière d'un sous-bois de pin maritime. DEA, Université de Paris-Sud, Orsay

Cohen Y, Fuchs M, Cohen S (1983) Resistance to water uptake in a mature Citrus tree. J Exp Bot 34, 451-460

Cohen Y, Kelliher FM, Black TA (1984) Determination of sap flow in Douglas fir trees using the heat pulse technique. Can J For Res 15, 422-428

Cohen Y, Moreshet S, Fuchs $M$ (1987) Changes in hydraulic conductance of Citrus trees following a reduction in wetted soil volume. Plant Cell Environ 10, 53-57

Cruiziat P, Granier A, Claustres JP and Lachaize $D$ (1989) Diurnal evolution of water flow and potential in an individual spruce: experimental and theoretical study. Ann Sci For 46 (suppl), 453s-457s

Decourt N, Lemoine B (1969) Tables de production provisoires pour le pin maritime dans le Sud-Ouest de la France. Ann Sci For 26, 344
Diawara A, Loustau D, Bergigier P (1991) Comparison of two methods for estimating the evaporation of a Pinus pinaster (Ait) stand: sap flow and energy balance with sensible heat flux measurements by an eddy covariance method. Agric For Meteor (sous presse)

Dixon MA, Grace J, Tyree MT (1984) Concurrent measurements of stem density, leaf and stem water potentials, stomatal conductance and cavitation of a sapling of Thuja occidentalis L. Plant Cell Environ 7, 615-618

Edwards WRN, Jarvis P, Landsberg JJ, Talbot $H$ (1986) A dynamic model for studying flow of water in single trees. Tree Physiol 1, 309324

El Hadj Moussa F (1989) Circulation de l'eau dans un peuplement de pins maritimes. Thèse, Université de Pau, $123 p$

Donner BL, Running SW (1986) Water stress response after thinning Pinus contorta stands in Montana. For Sci 32, 614-625

Fetcher N (1976) Patterns of leaf resistance to lodgepole pine transpiration in Wyoming. Ecology 57, 339-345

Granier A (1985) Une nouvelle méthode pour la mesure du flux de sève brute dans le tronc des arbres. Ann Sci For 42, 81-88

Granier A (1987) Evaluation of transpiration in a Douglas fir stand by means of sap-flow measurements. Tree Physio/ 3, 309-320

Granier A, Breda N, Claustres JP, Colin F (1989) Variation of hydraulic conductance of some adult conifers in natural condition. Ann Sci For 46 (suppl) 457s-460s

Granier A, Loustau D, Saugier B, Bergigier P (1990) Bilan hydrique de deux peuplements de pin maritime dans les landes : évaluation des flux des strates ligneuse et herbacée et de leur variabilité. Rapport ATP PIREN "Influence des couvertures pédologiques et végétales sur les bilans hydriques et minéraux du sol». (à paraître)

Granier A, Bobay V, Gash JHC, Gelpe J, Saugier B, Shuttleworth WJ (1990) Sap flow measurements of transpiration from a stand of maritime pine (Pinus pinaster Ait) in Les Landes Forest. Agric For Meteorol 51, 309319

Guyon JP, Kremer A (1982) Stabilité phénotypique de la croissance en hauteur et cinétique journalière de la pression de sève et de la 
transpiration chez le pin maritime (Pinus pinaster Ait). Can J For Res 12, 936-946

Hellkvist J, Parsby J (1976) The water relations of Pinus sylvestris. III. Diurnal and seasonal patterns of water potential. Physiol Plant 38, 61-68

Hinckley JM, Lassoie JP, Running SW (1978) Temporal and spatial variations in the water status of forest trees. For Sci Monogr 20, 1978

Jarvis PG, James GB, Landsberg JJ (1976) Coniferous forest. In: Vegetation and the Atmosphere (Monteith JL, ed) London Acad Press Vol II, p 171-240

Jarvis PG, Mc Naughton KG (1985) Stomatal control of transpiration : scaling up from leaf to region. Adv Ecol Res 15, 1-48

Katerji N, Durand $R$, Hallaire $M$, Perrier A (1982) Modèle de transfert de l'eau dans la plante et estimation des paramètres en conditions naturelles. CR Acad Sci Paris Sér III 294, 57-62

Kline JR, Reed KL, Waring RH, Stewart ML (1976) Field measurement of transpiration in Douglas-fir. J Appl Ecol 13, 273-284

Linder S, Benson M, Myers BJ, Raison RJ (1987) Canopy dynamics and Growth of Pinus radiata. I. Effects of irrigation and fertilization during a drought. Can J For Res 17, 1157-1165

Lopushinsky W (1986) Seasonal and diurnal trends of heat pulse velocity in Douglas-fir and ponderosa pine. Can J For Res 16, 814821

Loustau D, El Hadj Moussa FH (1989) Variability of stomatal conductance in the crown of a maritime pine (Pinus pinaster Ait). Ann Sci For 46 (suppl), 426s-429s

Monteith JL (1973) Principles of environmental physics. London, Edward Arnold
Pena J, Grace J (1986) Water relations and ultrasound emissions of Pinus sylvestris $L$ before, during and after a period of water stress. New Physiol 103, 515-524

Passioura JB (1984) Hydraulic resistance of plants - I constant or variable? Aust J Plant Physiol 11, 333-339

Running SW, Waring RH, Rydell RA (1975) Physiological control of water Flux in conifers. A Computer simulation model. CEcologia (Berl) $18,1-16$

Running FW (1980) Environmental and physiological control of water flux through Pinus contorta. Can J For Res 10, 82-91

Sandford AP, Jarvis PG (1986) Stomatal responses to humidity in selected conifers. Tree Physiol 2, 89-103

Schulze ED, Turner NC, Gollan T, Shackel KA (1987) Stomatal responses to air humidity and to soil drought. In: Stomatal Function (Zieger, Cowan, Farqhuar, eds) Stanford University Press, 311-321

Sharkey TD, Ogawa T (1987) Stomatal responses to light. In: Stomatal Function (Zieger, Cowan, Farqhuar, eds) Stanford University Press, 195-208

Snedecor GW, Cohran WG (1956) Méthodes statistiques. ACTA Paris, $649 \mathrm{p}$

Tyree MT, Dixon MA (1986) Water stress induced cavitation and embolism in some woody plants. Physiol Plant 66, 397-405

Tyree MT (1988) A dynamic model for water flow in a single tree: evidence that models must account for hydraulic architecture. Tree Physiol 4, 195-219

Tyree MT, Sperry JS (1988) Do woody plants operate near the point of catastrophic xylem dysfunction caused by dynamic water stress? Plant Physiol 88, 574-580 